INPLASY

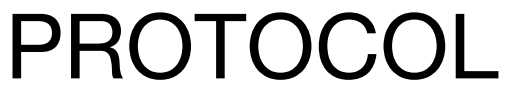

To cite: Sun et al. Survival benefit of surgical treatment added into systemic treatment for breast cancer liver metastases: A systematic review and meta-analysis. Inplasy protocol 202150063. doi:

10.37766/inplasy2021.5.0063

Received: 17 May 2021

Published: 17 May 2021

Corresponding author: Ling Xu

ling.xu@pkufh.com

Author Affiliation:

Peking University First Hospital.

Support: National Key R\&D Program.

Review Stage at time of this submission: Preliminary searches.

Conflicts of interest: None declared.

\section{Survival benefit of surgical treatment added into systemic treatment for breast cancer liver metastases: A systematic review and meta-analysis}

Sun, MS1; Liu, $\mathrm{HJ}^{2}$; Xu, L³.

Review question / Objective: Population: Breast cancer patients with liver metastasis; Intervention: Liver resection and/or radiofrequency ablation combined with systemic treatment; Control: Systemic treatment alone; Outcomes: Overall survival; Study design: Randomised controlled trial, cohort study, and case-control study.

Eligibility criteria: Studies comparing the prognosis of patients with breast cancer liver metastasis receiving systemic treatment combined with surgical treatment (surgical cohort) and those receiving systemic treatment alone (systemic cohort) will be included. The outcome of interest was overall survival, and hazard ratios (HRs) with corresponding 95\% confidence intervals (Cls) or survival curves should be provided. The papers should be accepted or published, and the full texts should be available in English. Noncomparative studies, duplicate studies, articles presented at meetings, review articles, conference abstracts, guidelines, case reports or series, and letters will be excluded. Comparisons of prognosis with a sample size of less than 20 in either the surgical cohort or the systemic cohort will be excluded because they are not statistically effective.

INPLASY registration number: This protocol was registered with the International Platform of Registered Systematic Review and Meta-Analysis Protocols (INPLASY) on 17 May 2021 and was last updated on 17 May 2021 (registration number INPLASY202150063).

\section{INTRODUCTION}

Review question / Objective: Population: Breast cancer patients with liver metastasis; Intervention: Liver resection and/or radiofrequency ablation combined with systemic treatment; Control: Systemic treatment alone; Outcomes: Overall survival; Study design: Randomised controlled trial, cohort study, and casecontrol study.

Condition being studied: Breast cancer liver metastasis (BCLM) is common and 
generally carries a poor prognosis. Systemic therapy has become the standard treatment, however, noting the curative potential of surgical treatment for colorectal and neuroendocrine liver metastases, some clinicians have advocated surgical treatment for a select group of patients with BCLM. There is growing evidence that surgical treatment may contribute not only to assessing histological information and the subtype of metastatic tumors, while also providing potential de-escalation of toxic systemic therapies and prolonging the time before more aggressive systemic therapy needs to be initiated. Nevertheless, experts have not reached a definitive consensus on this topic. The ESO-ESMO Guidelines for Advanced Breast Cancer note that surgical treatment for BCLM is considered in select patients, but the NCCN Guidelines do not recommend surgical treatment as an option. This is due to the lack of randomized data supporting the prognostic effect of surgical treatment and the inconsistent results of published retrospective studies. Most studies on this topic were retrospective, derived from small case series, and were highly susceptible to the effects of selection bias and confounding. Thus, an updated systematic review and meta-analysis is necessary to ascertain the prognostic value of surgical treatment for BCLM. Although previous systematic reviews supported surgical treatment for selected patients with BCLM because their survival outcomes appeared to be superior to systemic treatment alone, no meta-analysis of pooled data on this topic has been carried out. Our meta-analysis will the first pooled meta-analysis to confirm the survival advantage of surgical treatment for patients with BCLM. Therefore, our conclusion will the highest level of evidence to date on the prognostic benefits of surgical treatment for BCLM, since there are no relevant randomized controlled trials published until now.

\section{METHODS}

Search strategy: A systematic search of the Cochrane Library, Embase, and PubMed will be performed to identify relevant studies. The search strategy will be performed using the following medical subject headings and keywords: ("Breast Neoplasms"[Mesh] OR breast) AND ("Liver"[Mesh] OR liver OR hepatic) AND ("Neoplasm Metastasis"[Mesh] OR metastasis OR metastases OR metastatic) AND ("Hepatectomy"[Mesh] OR "Metastasectomy" [Mesh] OR "Radiofrequency Ablation"[Mesh] OR hepatectomy OR metastasectomy OR resection OR ablation OR ablative OR microwave OR surgery OR surgical).

Participant or population: Patients with breast cancer liver metastasis receiving systemic treatment combined with surgical treatment (surgical cohort) and those receiving systemic treatment alone (systemic cohort).

Intervention: Patients with breast cancer liver metastasis receiving systemic treatment combined with surgical treatment, including liver resection and radiofrequency ablation.

Comparator: Patients with breast cancer liver metastasis receiving systemic treatment alone.

Study designs to be included: Randomised controlled trial, cohort study, and casecontrol study.

Eligibility criteria: Studies comparing the prognosis of patients with breast cancer liver metastasis receiving systemic treatment combined with surgical treatment (surgical cohort) and those receiving systemic treatment alone (systemic cohort) will be included. The outcome of interest was overall survival, and hazard ratios (HRs) with corresponding 95\% confidence intervals (Cls) or survival curves should be provided. The papers should be accepted or published, and the full texts should be available in English. Noncomparative studies, duplicate studies, articles presented at meetings, review articles, conference abstracts, guidelines, case reports or series, and letters will be excluded. Comparisons of prognosis with a 
sample size of less than 20 in either the surgical cohort or the systemic cohort will be excluded because they are not statistically effective.

Information sources: A systematic search of the Cochrane Library, Embase, and PubMed will be performed to identify relevant studies. The references of relevant reviews and meta-analysis will also be manually searched for potentially relevant studies.

Main outcome(s): The main outcome is overall survival, and the hazard ratios (HRs) with corresponding $95 \%$ confidence intervals (Cls) or survival curves should be provided.

\section{Additional outcome(s): None.}

Data management: Two authors will independently scan the titles and abstracts from the studies identified in the electronic search. Relevant papers will be further identified through perusing full texts. Disagreements will be resolved by discussion and consensus between authors. Two researchers will review each article by a structured list and extract data into a database independently. Disagreements in data extraction will be resolved by discussion. Data with the following items will be extracted: (1) Characteristics including first author, country, publication year, sample size, age of patients, approach of surgical treatment, study years, study design, study center, size and number of liver metastases, and covariates included in matching. (2) The hazard ratio (HR) for overall survival between the surgical cohort and the systemic cohort. When the study reports the HRs, we will extract them directly. When the study reports the HRs of both univariate analysis and multivariate analysis, the HRs of multivariate analysis will be extracted.

Quality assessment / Risk of bias analysis: The Newcastle-Ottawa scale (NOS) will be used to evaluate the methodological quality of the studies. The scale has three parts: Patient selection, comparability, and outcome. Two reviewers will appraise the quality of studies independently. Disagreements will be solved by consensus. The scale changes from 0 to 9 stars, and studies with a score $\geq 7$ stars could be deemed as high quality. The variables related to liver metastasis (e.g. time between breast cancer and liver metastasis diagnosis, number of liver metastasis, maximum diameter of liver metastasis) will be selected as the most important controlling factor in "comparability", because previous studies confirmed them as key survival predictors for patients with breast cancer liver metastasis and these characteristics significantly influenced the clinical decision-making of treatment for these patients.

Strategy of data synthesis: The R software version 3.6.3 (http://www.r-project.org) will be adopted to perform the meta-analysis. Forest plots for comparison of overall survival between surgical and systemic treatment will be performed. The pooled analysis of the HRs of the included studies will also be performed. The $I^{2}$ statistic will be utilized to evaluate the heterogeneity among the studies. The random effects model will be adopted if $\mathrm{I}^{2}$ was $>50 \%$, otherwise the fixed-effects model will be utilized. Potential publication bias will be determined by a funnel plot and assessed by the Begg's test and Egger's test. In all cases, $P$ value $<0.05$ will be considered statistically significant.

Subgroup analysis: The surgical treatment in studies was performed in different ways, some of which were liver resection while others were radiofrequency ablation. We will therefore conduct subgroup analyses by categorizing the types of surgical treatment to improve the reliability of the conclusions.

Sensitivity analysis: To overcome the effects of selection bias, some studies have managed to balance the baseline characteristics of surgical and medical cohorts with some methods, including paired design and propensity score matching. In our study, we will combine the 
matched and unmatched cohorts separately to verify our conclusions.

Language: English.

Country(ies) involved: China.

Keywords: surgical treatment; breast cancer; liver metastases; survival; liver resection; radiofrequency ablation.

Contributions of each author:

Author 1 - Ming-Shuai Sun will design the study, collect and analyze the data, conceive the idea for the paper, and draft the manuscript.

Email: sun_mingshuai@126.com

Author 2 - Hong-Jin Liu will collect and analyze the data, conceive the idea for the paper, and draft the manuscript.

Email: liuhj_pkufh@163.com

Author 3 - Ling Xu will help design the study, conceive the idea for the paper, and draft the manuscript.

Email: ling.xu@pkufh.com 